9

\title{
Environmental modeling and simulation - applications and future requirements
}

\author{
R. Grützner \\ University of Rostock \\ Department of Computer Science, \\ Albert-Einstein-Str. 21, D-18059 Rostock, Germany \\ Tel.:+49(381)4983369, Fax: +49(381)4983426 \\ e-mail:gruet@informatik.uni-rostock.de
}

\begin{abstract}
This paper gives a short report on the state of the art in the method of problem solving by modeling and simulation used in environmental protection. Application fields, problems, and future developments will be discussed. Requirements and new approaches to the software tools for model and experimental description, simulation system architectures for parallel and distributed simulation experiments will be represented.
\end{abstract}

\section{Keywords}

modeling, simulation experiments, simulation system architecture, environmental applications

\section{BASE DEFINITION}

Up to now the state of the environment has been changed by human activities in a dramatic and dangerous way. This situation must be stopped as soon as possible. Therefore, we need innovative theoretical and practical scientific approaches. The urgency of the problem poses a challenge to computer science and particularly to environmentally oriented informatics. Important methods and tools are modeling and simulation. Modeling and simulation is a problem solving method where problems are solved not by experimentation with real world systems but by experiments using models of real world objects. Using this method, the main task is to develop adequate models of the real world systems which we want to examine. If a simulation model adequately represents the important aspects of the real system, selected for investigations, a 
conclusion is then permitted from the simulation results to the behaviour of the real system. Therefore, modeling and simulation is a very important method in practical and theoretical environmental research and protection.

To solve a given problem every model pursues a special aim - the model or simulation aim which defines the scope of the model. Only these components of a real system are mapped in a model which are significant for the defined model aim. That means modeling is a process of abstraction and generalization neglecting all unimportant real world system components (without any contribution to the model aim).

Simulation models are abstract mathematical descriptions of system relations, the relations between the states of a system. For descriptions model definition languages (e.g. DYMOLA) or simulation languages (e.g. SIMULA, ACSL) are used.

Depending on the value scope of the state variables given, we distinguish models or systems as continuous, discrete or combined. Models are called continuous if the variables of the system possess values from an intervall, discrete if system variables assume discrete values. In combined models system variables assume discrete and continuous values.

To get simulation results, we have to experiment with models. In an experiment computation methods are applied to models (models are executed by a method). Computation methods or short methods are computation algorithms. A base method is the simulation method which controls the time-dependent dynamical behaviour of the system state. Other examples of methods at a higher level are optimization of parameters or system trajectories, sensitivity analysis, and Monte-Carlo methods (stochastic changing of parameters or inputs).

Experiments require an appropriate experimental description to define the kind of problem solving needed. If model and experimental descriptions are separated, we get an important base for a multiple use of models.

Essential software systems for modeling and simulation activities are simulation systems. A simulation system supports the user during the phases of model building and description, the experiment definition process, the execution and supervision of simulation experiments, the analysis and visualization of simulation results, the managment of models, data, and experimentation methods.

\section{MODELLING AND SIMULATION OF ENVIRONMENTAL SYSTEMS}

Modeling and simulation in the environmental domain today is used to determine the dynamic behaviour of systems in order to make decisions or to test the impact of human actitivities (e.g. the use of solar energy for heating, construction, production processes, traffic and logistic planning and decisions) on the environment. Further questions will be added in the near future because of the increasing complexity of the systems: the assessment of alternative decisions and activities, the examination of sceneries, the analysis of eco-economical and of socio-economical systems. Additional future assignments would be the evaluation and optimization of the whole life-time cycle of products related to their impacts on the environment. The life-time cycle of a product consists of its production, use, and finally, the recycling process.

To solve these assignments, new experimentation approaches, simulation methods, computation methods, and software tools are needed. Several of the application fields and some software architectures for modeling and simulation will be represented in the following. 


\subsection{Problems of Modeling Environmental Systems}

The modeling and simulation of environmental systems requires the consideration of a lot of special system properties which complicate the analysis considerably.

We have to consider different groups of problems. One group results from the complexity of the environmental systems (e.g. the biological, ecological, economical properties) and the other group results from the degree of the user's qualification in managing these problems (using the models, storing and preparation of data, interpretation of results and, the making correct decisions).

Problems of modeling environmental systems:

- there is a lack of theoretical knowledge

- causal relations between system components are frequently unknown

- most of the real systems can be observed by experiments, but frequently there isn't any possibility for controlled experiments

- ecological systems very often possess long reaction times (up to years or centuries) and in contrast, other components possess very short reaction times

- system complexity is high because of the strong relations between the system components

- nature is an open self-organizing system with adaptable components

- inaccurate data and ill-defined system components exist

- there is an increasing set of data: spatial data and spatial data with time dependence.

- often it isn't possible to define exact and unique optimization aims for ecological and environmental systems

- the model description must be realized by analytical and non analytical (rule based) methods.

Another group of problems result from the different levels of knowledge by users in the field of environmental protection. There are highly qualified specialists without any knowledge in the field of simulation and in the use of complex software systems. This situation requires software with excellent user interfaces and user support components for the whole model-life-time cycle. Unfortunately, we can't very often find simulation software which possess only some of these expected support components. Such systems are still in research.

\subsection{Fields of Application}

The most important tasks in the field of environmental modeling and simulation today are still the determination and analysis of the behaviour of environmental systems, that means the computation of the time dependent dynamic behaviour of state variables.

Some applications of modeling and simulation are given below. On the one hand, undesired secondary effects of human activities (e.g. emissions of harmful substances of production processes) can appear. In such a situation, the secondary effects to the environment are to minimize. On the other hand, actions of environmental protection are to optimize. These are investigations of the questions: how does a process work? or which effects are released in the environmental system by a special input?

In the environmental domain, simulation models are especially used in four fields: emission computation (air, water, ground pollution), process control, groundwater - economical and flow investigations, and ecosystem research. 
But further applications become more and more important: e.g., models on the use and balance of resources (e.g. water, ground, materials, energy, food); models of the carrying capacitiy and of loading limits of ecological systems with an input, which has been caused by human activities; quality models; product-life-time-cycle models and ecobalances; socio-economic models; combined tasks: the use of simulation and optimization methods or experiments consisting of coupled economical and ecological models.

\section{Models of pollution extension}

An important task in environmental protection is the computation of immission loads caused by emission processes. Most environmental problems are caused by the emission of harmful and dangerous substances which are spreaded in air, water, and on the ground. The computation of the expansion of radiation, noise, vibrations, chemical reactions of substances and their effects on human life are of high interest today.

The primary targets of extension models are:

- computation of pollution values at geometrical points where we don't have any measurement results

- detection of pollution sources by simulation experiments

- supporting position planning and permission processes for objects with emissions (e.g. of factories, production processes, traffic projects, buildings)

- determination of reactions to accidents (e.g. of chemical processes, of oil transports).

An overview of the state of the art is given by (Zannetti,1990), (Wrobel,1991), (Melli,1992). Models are urgently needed in the future for the computation of pollution and distribution in the micro scale range (near the ground). For example, there are problems regarding the distribution of auto exhaust in street canyons depending on traffic density and kind of formation of buildings near the street.

\section{Water models}

The pollution and the increased use of water based on constant water resources has produced evident environmental damage all over the world. This causes a high importance of water models. There are three main groups of models:

- investigations of the influence of structures, buildings and other human activities on the amount and flow of groundwater

- investigations of the levels of groundwater depending on irrigation, draining, and soil erosion.

- waste water models and water cleaning processes.

An important future assignment will be the modeling and simulation of the relations between vegetation and groundwater levels .

\section{Models for process control simulation}

Models are used to evaluate the consumption of recources of technical processes and the emission of these processes. The primary target is the minimizing of the environmental influence. Models are used during the planning phase and the model supported process control to get an optimal process behaviour. Examples of process models are given by (Keller, 1994), (Tuma, 1994), (Früh, 1989). 


\section{Modeling of ecosystems}

The two fields of environmental protection and ecosystems are strongly connected. But today a broad use of simulation is still difficult. Simulation is used only in selected parts, e.g. community ecology in water, ground, forest; nitrogen balance; steadiness and elasticity of ecosystems - that means the capability of taking up harmful substances without any effects on the ecological balance; effects of chemical substances in ecological systems.

More than in other applications, modeling and simulation is an interdisciplinary task. The following part of this paper will refer to the modeling and simulation of ecological systems connected with environmental protection.

\subsection{Problems and Expected Results}

Modeling and simulation cause some problems when using existing simulation systems and simulation software tools. The main problems are:

- it is necessary to get a better understanding of the dynamic behaviour of the systems by using structure edaquate models

- missing system support for investigations of human processes in such a way, that damages to the environment will be at a minimum

- handling the increasing system complexity (based on extensions of system borders and/or detailed system investigations) by modular-hierarchical modeling concepts

- development of comfortable user interfaces of software tools for simulation experiments.

An improvement in the present situation can be achieved on the one hand by the building of more precise models (e.g. of structure adequate models, (Bossel,1994)) and on the other hand by the development of better simulation software. We will explain the last point below.

The complexity of environmental systems demands a model description so that the method of description can be adapted to knowledge about the system. Models consisting of different described modules may be called heterogenous. For instance one modul of the model may be described by differential equations and other moduls by rules, difference equations, higher Petri-nets (ecocontroling models), or other tools. The simulation systems must be able to simulate such heterogenous models. That means, that dependent on the description method of a module appropriate simulation methods must be used. The structure of the simulation system must be changed to realize simulation runs of heterogenous models.

An effective experimentation requires control of experiments either interactive or by an experiment description. Experimental control means to fix the methods, sequence of use, and data to solve a problem in a simulation experiment. Methods are used for optimization, sensitivity analysis, investigation of transient states, steady-state detection, visualization, and so on.

\section{MODERN STRUCTURES OF SIMULATION SYSTEMS}

The following considerations are restricted to the modeling and simulation of ecological problems, that means the consideration of combined systems which are described by systems of ordinary differential equations of first order (system state equations).

Computer science can support the modeling and simulation in three important tasks: 
- building and managment of models and experiments

- supervision of simulation experiments by adequate simulation system architectures

- simulation result analysis and visualization.

The first and second points are investigated in our projects SAME and SAMEC (advanced by the Deutsche Forschungsgemeinschaft), (Grützner,1993).

\subsection{Models and Modeling}

One possibilty in handling the system complexity and to guarantee the multiple use of models is the separation of model and experiment description (Schwarze, 1990), (Zeigler, 1986), (Zeigler,1989). Up to now this has been realized in only a few systems.

Separation means that a model represents only the structural description of a class of systems. All of the values of parameters, of initial state variables, and system input variables are defined in the experimental description belonging to the model. Aside from the values an experimental description also defines the operations (or methods) which are applied to the model (e.g. optimization of parameters) during the experiment's execution. The experiments control the model and the method execution during the simulation process.

But there is a disadvantage in the separation because we need two languages, which a user must know; one language for the model and another one for the experimental description.

Present model description languages are simulation languages belonging to a special simulation system. This is a disadvantage because we are unable to exchange models between different simulation systems and the user has to learn another simulation language if he wants to use a new simulation tool. For the considered field of application, it seems important for the future to look for a general modeling language independent of any simulation system. Such a general language will allow a better model exchange and system understanding. To do a simulation currently, the model description would have to transform into the language used by the system. Such an approach has been used successfully by electrical and electronical model descriptions. The proposed language VHDL-A will be standardized soon (Bausch-Gall, 1994).

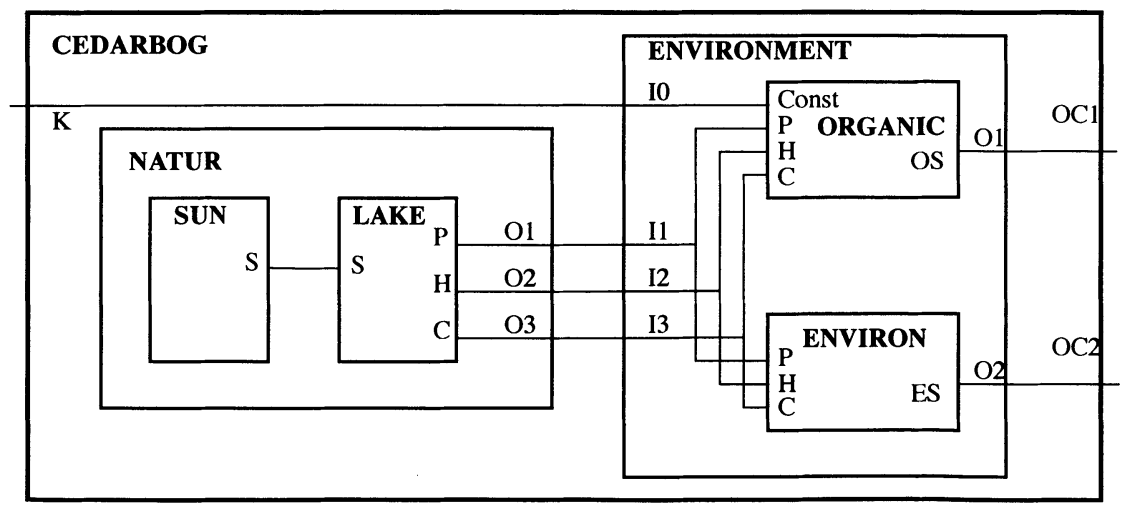

Figure 1 Graphical description of the modular-hierarchical model CEDARBOG. 


\section{Modell Description}

The models in the environmental domain are modular-hierarchical (a second concept to handle the system complexity) and of a variable structure. The systems are decomposed into base objects. To find a solution to an existing problem, a further decomposition of a base object isn't necessary. Base objects contain the equations for the dynamic behaviour of the system. Coupled base objects are called submodels. These can be coupled again to get submodels at a higher level. So a model can be used as a submodel in another model. This means object oriented modeling, where a base object represents a real system object.

It is important for users to have a model description language and a graphical modeling tool at the same time. A mix of both descriptions in one model should be allowed.

The graphical model description of a simple example CEDARBOG of a silting-up process in a lake see Figur 1 and the description by the language SAME-MDL see Figur 2. The model consists of two submodels, NATURE and ENVIRONMENT, which consist of two base objects. SUN defines the energy input by the sun, base object LAKE defines the state of plants $(\mathrm{P})$, of herbivorous objects $(\mathrm{H})$, and of carnivorous objects $(\mathrm{C})$. The base object ORGANIC

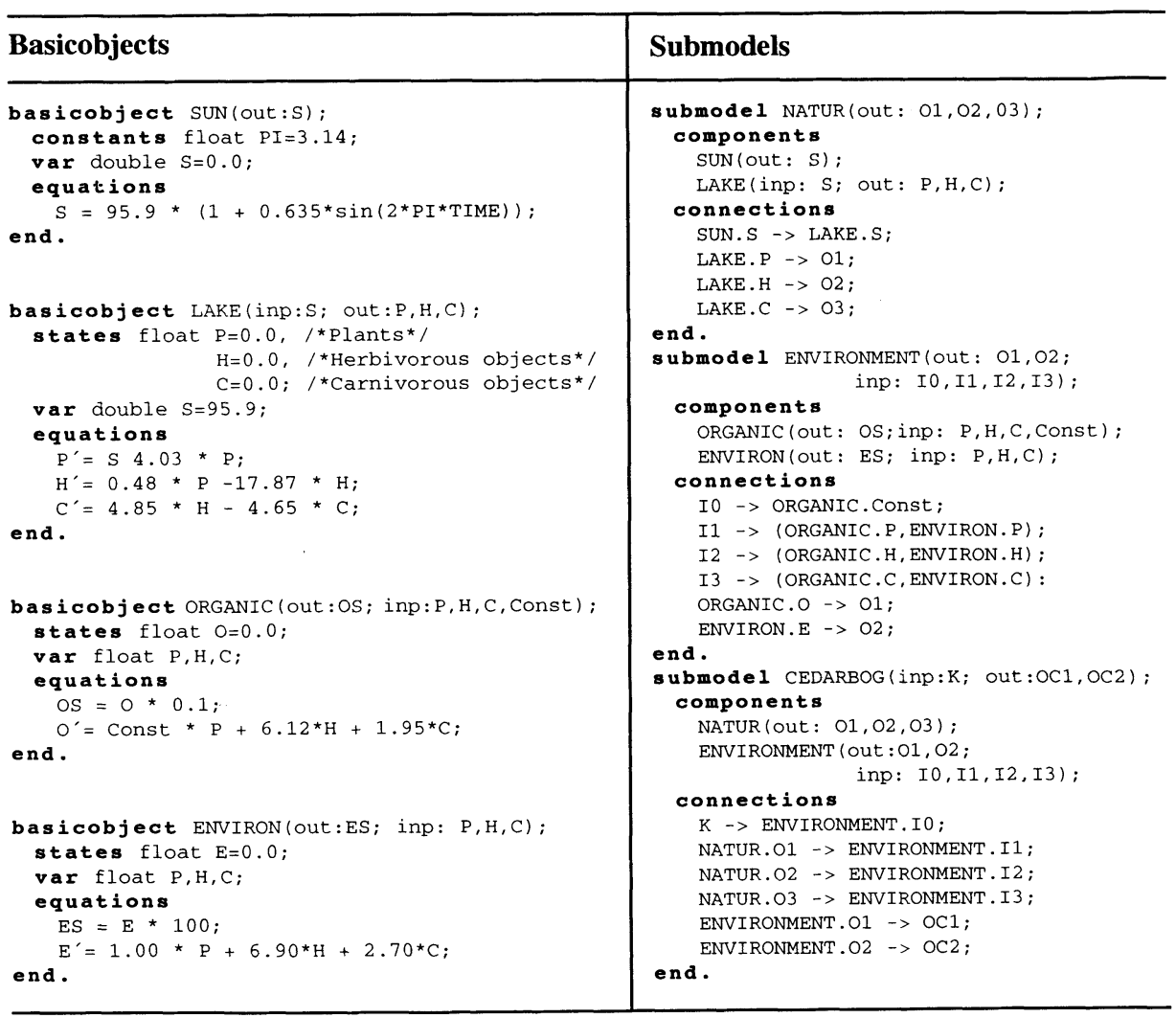

Figure 2 Model description of CEDARBOG using SAME-MDL, (Dimitrov,1994). 
models the sediment process and ENVIRON the energy output to the environment.

Because of the above mentioned problems in environmental modeling, it's important to allow the use of different mathematical descriptions of the dynamic behaviour of the base objects, e.g. by differential and difference equations, rule based approaches, fuzzy rules, and neuronal net model components. Up to now this hasn't been realized by SAMEC.

\section{Experimental Description}

The proposed procedural experiment description language (Dimitrov,1994) is based on the frame concept. A frame is a structuring element for an experiment also to define subexperiments and a parallel experiment execution. Subexperiments can be described depending on additional conditions. One example of such an experiment description is given by Figure 3 . This is an experiment with the model CEDARBOG for two parallel experiments using this modell. CEDARBOG may be used with two different parameter sets.

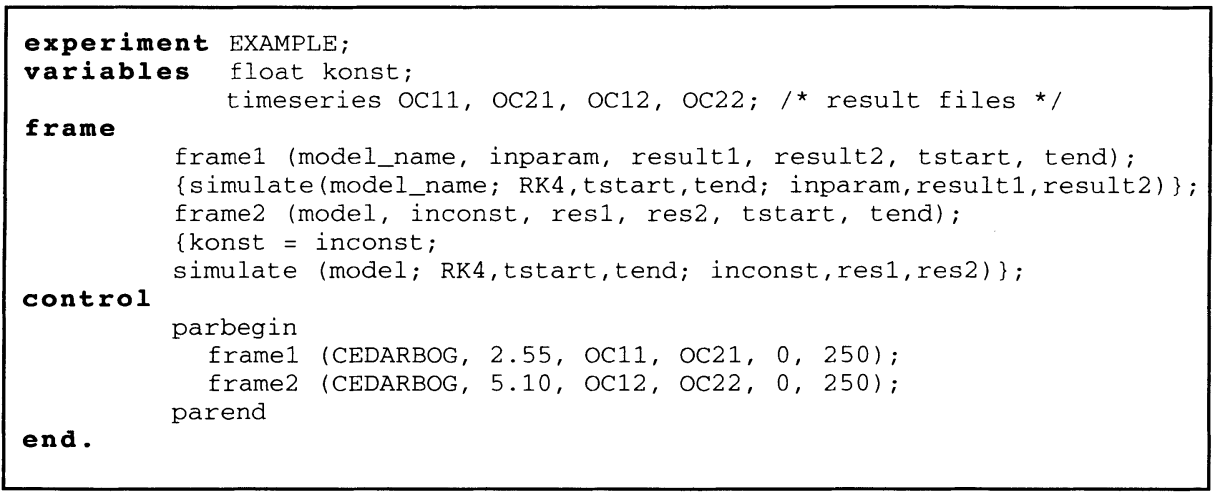

Figure 3 Description of two parallel experiments with the model CEDARBOG.

\subsection{Simulation Systems}

Let us consider simulation systems for ecological systems. The architecture of simulation systems is affected by the following requests:

- interactive use in all working phases and user friendly interfaces

- support of the users in the building and managment processes of models and experiments

- parallel and distributed execution of experiments

- use of innovative methods: genetical algorithms, fuzzy set and fuzzy rule based concepts, neuronal nets

- coupling of simulation systems with information systems (geo-information systems).

\section{Parallel simulation}

Based on the modular-hierarchical model concept, we have realized a system architecture for parallel and distributed simulations of base objects. The system of algebraic-differential equations is separated into subsystems, the equations of the base objects. For the parallel simulation 
of the base objects special integration algorithms have been used (Schäfer,1994), (Werner,1995). Another iterative algorithm for parallel simulation, which uses the classical numerical integration methods for base objects, is proposed by (Singh,1986). But in contrast to the first algorithms the iterative solution is inaccurate.

To control the couple relation between the base objects and the submodels special coordinator routines are introduced. A coordinator realizes the data exchange between the model components and some functions for the integration process. The external structure of the model is in this architecture equivalent to the internal structure. Some results of measurements neglecting the communication overhead indicate an evident speed up (Werner,1995).

\section{Parallel experiments}

An experiment description also allows a parallel experiment realization. Each experiment is then controlled by its own experiment process. Parallel experiments are especially important (high speed up) for parameter and trajectory optimization, sensitiveness investigations, and other multiple simulation runs.

The communication between processes are based on the PVM concept (Parallel Virtual Machine). A special communication protocol of simulation system processes (PSP) has been defined for implementation (Nekien,1995). The implementation has been realized at an UNIX workstation net.

\subsection{Coupling of Simulation Systems with other Software Tools}

Modeling and Simulation of environmental systems means working with a lot of data (e.g. input, geographical, environmental, result data). For an effective simulation all data must be provided automatically from different data bases (e.g. geographical information systems, environmental information systems). The access to the data supposes a coupling of simulation systems and data bases. The data which are used in the simulation experiments must be defined in the experiment description in such a way, that no manual actions by users are necessary. The access description to data bases in experiments must be possible without any knowledge of internal data base structures.

\section{REFERENCES}

Bausch-Gall,I. (1994) Modellaustausch zwischen Simulationsprogrammen. in Fortschritte der Simulationstechnik, Bd. 9, ASIM 9. Symposium (ed. G. Kampe \& M. Zeitz), Vieweg, Stuttgart. Bossel, H. (1994) Understanding Dynamic Systems: Shifting the Focus from Data to Structure, in 8. Symposium Informatik für den Umweltschutz (ed. L. M. Hilty, A. Jaeschke, B. Page, A. Schwabl .), Bd.1, Metropolis Verlag, Marburg, 63-75.

Dimitrov, E. (1994) Modell- und Experimentbeschreibungen für Umweltproblemstellungen und ihre Implementation . Preprint No. CS-01-94, University of Rostock, Dept. of Computer Science, WG Modeling/Simulation.

Früh, K. F. (1989) Informationsorientierte Prozeßleittechnik für die Chemische Industrie. Automatisierungstechnische Praxis atp, 31(7), 301-305.

Grützner, R. (1993) Abschlußbericht zum Projekt SAME. University of Rostock, Dept. of Computer Science, Report of the WG Modeling/Simulation 
Keller, H. B., Kugele, E., große Osterhues, B. (1994) Einsatz neuronaler Netze im Umweltbereich am Beispiel der Müllverbrennung. in 8. Symposium Informatik für den Umweltschutz (ed. L. M. Hilty, A. Jaeschke, B. Page, A. Schwabl .), Bd.1, Metropolis Verlag, Marburg, 405414.

Melli, P., Zannetti, P. (1992) Environmental Modelling. Computational Mechanics Publications Southampton.

Nekien, T. (1995) The communication protocol in the SAME-simulator. University of Rostock, Dept. of Computer Science, WG Modeling/Simulation, in publishing.

Schäfer, P. (1994) Parallele Verfahren zur Simulation modularer technischer Systeme. in ASIM 9. Symposium (ed. G. Kampe \& M. Zeitz), Vieweg, Stuttgart.

Schwarze, G. (1990) Digitale Simulation. Akademie Verlag, Berlin.

Singh, M. G., Allidina, A. Y., Daniels, B. K. (1986) Parallel Processing Techniques for Simulation. Plenum press, New York, London.

Tuma, A., Haasis, H. D. (1994) Real Time Production Scheduling of Environmental Integrated Production Systems - A Comparision of Selected Knowledge-Based Methods and Maschine Learning Algorithms. in 8. Symposium Informatik für den Umweltschutz (ed. L. M. Hilty, A. Jaeschke, B. Page, A. Schwabl), Bd.1, Metropolis Verlag,Marburg, 371-8

Wrobel, L. C., Brebbia, C. A. (1991) Water Pollution: Modelling; Measuring and Prediction. Computational Mechanics Publication, Southampton.

Werner, G.(1995) Vergleichende Analyse von parallelen Simulationsmethoden zur Ausführung komplexer Modelle. University of Rostock, Dept. of Computer Science, Diplomarbeit.

Zannetti, P. (1990) Air Pollution Modelling. Computational Mechanics Publication, Southampton.

Zeigler, B. P., Oren, T. I. (1986) Multifacetted Multiparadigm Modelling Perspectives: Tools for the 90's. in Winter Simulation Conference. SCS Publications, Washington , 708-712.

Zeigler, B. P., Zhang, G. (1989) The Entity Structure: Knowledge Representation for Modeling Design. in Artificial Intelligence, Simulation, and Modeling (ed. L. E. Widman, K. A. Loparo, N. R. Nielsen), John Wiley\&Sons, New York, 47 - 73.

\section{BIOGRAPHY}

After a study of applied aerodynamics and an activitiy of seven years in the industry follows an activity as research worker at the Humboldt University of Berlin, fields of modeling and simulation, computer performance analysis, and operating systems. Since 1989 professor for modeling/simulation at the University of Rostock. Working fields: architecture of simulation systems, parallel simulation, user interfaces, application of simulation in environmental research. 